\title{
Performance Analysis of Wireless Systems in the Presence of $k-\mu$ Short Term Fading, Gamma Long Term Fading and $\mathrm{k}-\mu$ Cochannel Interference
}

\author{
Dragana Krstić, Mihajlo Stefanović, \\ Vesad Doljak \\ Faculty of Electronic Engineering, \\ University of Niš, \\ Niš, Serbia \\ dragana.krstic@elfak.ni.ac.rs \\ Danijela Aleksić \\ College of Applied Technical Sciences, \\ Niš, Serbia
}

\author{
Muneer Masadeh Bani Yassein \\ Department of Computer Science \\ Jordan University of Science and Technology \\ Irbid, Jordan \\ masadeh@just.edu.jo
}

\author{
Milan Gligorijević \\ Criminalistics-Police Academy, \\ Belgrade, Serbia
}

\begin{abstract}
Wireless communication system operating over Gamma shadowed multipath fading channel in the presence of cochannel interference subjected to small scale fading is delineated in this paper. Desired signal experiences Gamma long term fading, $k-\mu$ short term fading and cochannel interference affected by $k-\mu$ multipath fading. In interference limited environment, the signal to interference ratio (SIR) for proposed wireless communication system can be calculated as the ratio of product of square rooted Gamma random variable and $k-\mu$ random variable and $k-\mu$ random variable. The probability density function (PDF), moments and moment generating function of signal to interference ratio will be calculated as expressions in the closed form. The impact of Rician factor of desired signal and Rician factor of cochannel interference on moments will be analyzed and discussed.
\end{abstract}

Keywords-cochannel interference; Gamma shadowing; $k-\mu$ fading; moments; moment generating function; probability density function

\section{INTRODUCTION}

The $\mathrm{k}-\mu$ short term fading, Gamma long term fading and $k-\mu$ cochannel interference degrade the outage probability and average symbol error probability of wireless communication system [1][2]. The $\mathrm{k}-\mu$ statistical model can describe small scale desired signal envelope variation in line of sight multipath fading environment where signal propagates with two or more clusters [3]. The $\mathrm{k}-\mu$ distribution is described with two parameters, $k$ and $\mu$ [4]. The parameter $k$ is Rician factor and defined as the ratio of dominant component power and scatter components power. The parameter $\mu$ is in relation with the number of clusters in propagation environment.

The $\mathrm{k}-\mu$ distribution is general distribution and Rayleigh, Rician and Nakagami-m distributions can be derived from $\mathrm{k}-\mu$ distribution as special cases [5]. By using for $k=0$, the $k-\mu$ distribution reduces in
Nakagami- $m$ distribution and for $\mu=1$, Rician distribution is derived from $\mathrm{k}-\mu$ distribution. Rayleigh distribution is obtained from $\mathrm{k}-\mu$ distribution by setting $\mathrm{k}=0$ and $\mu=1$. When $k$ goes to infinity, the $\mathrm{k}-\mu$ multipath fading channel becomes no fading channel.

Long term fading can be described by using lognormal distribution or Gamma distribution. When signal envelope average power is modeled by using the Gamma distribution, the expressions for outage probability and bit error probability can be derived in the closed form.

There are a lot of papers in open technical literature considering performance of wireless communication system in the presence of long term fading and short term fading and performance of wireless communication system in the presence of short term fading and cochannel interference.

In papers [6]-[9], the second order performance measures of macrodiversity system with macrodiversity selection combining receiver and two microdiversity maximal ratio combining receivers operating over shadowed multipath fading channels are evaluated. In [10]-[12], wireless communication systems with selection combining receiver working over multipath fading channels in the presence of cochannel interference subjected to short term fading are analyzed. For this model, probability density function, cumulative distribution function, moments, outage probability and bit error probability are calculated.

In this paper, wireless communication system operating over multipath fading and the presence of cochannel interference subjected to long term fading and short term fading is estimated. Desired signal is subjected to $\mathrm{k}-\mu$ short term fading and cochannel interference experiences Gamma long term fading and $\mathrm{k}-\mu$ short term fading. In interference limited environments, the ratio of desired signal envelope to 
cochannel interference envelope is important performance measure. For proposed model, signal to interference ratio can be calculated as ratio of product of square rooted Gamma random variable and the $k-\mu$ random variable and the $k-\mu$ random variable. The probability density function, moment generating function and moments of proposed ratio are calculated as expressions in the closed form.

\section{Performance of OutPut Signal to INTERFERENCE RATIO}

\section{A. Probability Density Function of Output Signal Envelope to Interference Envelope Ratio}

The output signal to interference ratio (SIR) is an important entity in communications engineering which indicates the quality of a link between transmitter and receiver in a multi transmitter-receiver environment.

By definition, the noise is the unwanted signal that contaminates the original signal causing false decision on the information contained by the original signal at the receiver. There are two types of noise according to their source. The noise is said to be intrinsic if it is generated in the communication devices such as the thermal, the shot and the $1 / \mathrm{f}$ noise generated in the resistive elements inside the electronics. This is because of the statistical nature of the electronic conduction in the element of the circuits. The second type of noise is the extrinsic noise which arises from interference of the other signal sources that might be exiting in the same space and time of the original signal. The intrinsic noise is simply called noise and the extrinsic noise is called simply interference signal [13].

So, at any point of the path between the transmitter and the receiver there is a value for the wanted signal power, called $\mathrm{S}$ and there is noise $\mathrm{N}$ and interference signal power I. The most important point is the point of the decision on the information value of the symbol in the receiver. Ideally, at this point theoretically speaking one has to make $\mathrm{N}=0$ and $\mathrm{I}=0$. The interference can be eliminated if it is known. If it is unknown, one can measures it and subtract it from the signal. It is the correlation between signal and interference that cannot be eliminated. The intrinsic noise can be reduced by using low noise devices and matched filters. Since the received signal $r=S+N+$ $\mathrm{I}$, it is observed the signal to noise plus interference ratio $\mathrm{S} /[\mathrm{I}+\mathrm{N}]$ at the input of the decision device in the receiver.

The SINR is defined as the power of a certain signal of interest divided by the sum of the interference power (from all the other interfering signals) and the power of some background noise. If the power of noise term is close to zero, then the SINR reduces to the signal-to-interference ratio (SIR). Conversely, zero interference reduces the SINR to the well known signal-to-noise ratio (SNR). SINR is commonly used in wireless communication as a way to measure the quality of wireless connections.

If the level of interference is significantly higher than the level of noise in the channel, we will also use SIR for further analysis of wireless systems.
The ratio of product of square rooted Gamma random variable and $\mathrm{k}-\mu$ random variable, and $\mathrm{k}-\mu$ random variable is:

$$
w=\frac{x \cdot y}{z} .
$$

The random variable $x$ is:

$$
x=x_{1}^{1 / 2}, x_{1}=x^{2},
$$

where $x_{l}$ has Gamma distribution:

$$
p_{x_{1}}\left(x_{1}\right)=\frac{1}{\Gamma\left(c_{1}\right) \beta_{1}^{c_{1}}} x_{1}^{c_{1}-1} e^{-\frac{1}{\beta_{1}} x_{1}}, x_{1} \geq 0
$$

Probability density function of $x$ is:

$$
p_{x}(x)=\left|\frac{d x_{1}}{d x}\right| p_{x_{1}}\left(x^{2}\right)=\frac{2}{\Gamma\left(c_{1}\right) \beta_{1}^{c_{1}}} x^{2 c_{1}-1} e^{-\frac{1}{\beta_{1}} x^{2}}, x \geq 0
$$

The random variable $y$ follows $k-\mu$ distribution [4]:

$$
\begin{gathered}
p_{y}(y)=\frac{2 \mu_{1}\left(k_{1}+1\right)^{\frac{\mu_{1}+1}{2}}}{k_{1}^{\frac{\mu_{1}-1}{2}} e^{k_{1} \mu_{1}} \Omega_{1}^{\frac{\mu_{1}+1}{2}}} y^{\mu_{1}} \cdot e^{-\frac{\mu_{1}\left(k_{1}+1\right)}{\Omega_{1}} y^{2}} \cdot I_{\mu_{i}-1}\left(2 \mu_{1} \frac{\sqrt{k_{1}\left(k_{1}+1\right)}}{\Omega_{1}} y\right)= \\
=\frac{2 \mu_{1}\left(k_{1}+1\right)^{\frac{\mu_{1}+1}{2}}}{\frac{\mu_{1}-1}{k_{1}^{2}} e^{k_{1} \mu_{1}} \Omega_{1}^{\frac{\mu_{1}+1}{2}}} \cdot \sum_{i=0}^{\infty}\left(\mu_{1} \sqrt{\frac{k_{1}\left(k_{1}+1\right)}{\Omega_{1}}}\right)^{2_{i}+\mu_{1}-1} \frac{1}{i_{1} ! \Gamma\left(i_{1}+\mu_{1}\right)} . \\
\cdot y^{2 i_{1}+2 \mu_{1}-1} \cdot e^{-\frac{\mu_{1}\left(k_{1}+1\right)}{\Omega_{1}} y^{2}}, y \geq 0 .
\end{gathered}
$$

Random variable $z$ has also $k-\mu$ distribution [4]:

$$
\begin{gathered}
p_{z}(z)=\frac{2 \mu_{2}\left(k_{2}+1\right)^{\frac{\mu_{2}+1}{2}}}{k_{2}^{\frac{\mu_{2}-1}{2}} e^{k_{2} \mu_{2}} \Omega_{2}^{\frac{\mu_{2}+1}{2}}} \sum_{i_{2}=0}^{\infty}\left(\mu_{2} \sqrt{\frac{k_{2}\left(k_{2}+1\right)}{\Omega_{2}}}\right)^{2 i_{2}+\mu_{2}-1} \frac{1}{i_{2} ! \Gamma\left(i_{2}+\mu_{2}\right)} . \\
\cdot z^{2 i_{2}+2 \mu_{2}-1} \cdot e^{-\frac{\mu_{2}\left(k_{2}+1\right)}{\Omega_{2}} z^{2}}, z \geq 0
\end{gathered}
$$

Probability density function of $w$ is:

$$
\begin{aligned}
& p_{w}(w)=\int_{0}^{\infty} d y \int_{0}^{\infty} d z \cdot \frac{z}{y} \cdot p_{x}\left(\frac{w z}{y}\right) p_{y}(y) p_{z}(z)=\frac{2}{\Gamma\left(c_{1}\right) \beta_{1}^{c_{1}}} w^{2 c_{1}-1} . \\
& \cdot \frac{2 \mu_{1}\left(k_{1}+1\right)^{\frac{\mu_{1}+1}{2}}}{k_{1}^{\frac{\mu_{1}-1}{2}} e^{k_{1} \mu_{1}} \Omega_{1}^{\frac{\mu_{1}+1}{2}}} \sum_{i_{1}=0}^{\infty}\left(\mu_{1} \sqrt{\frac{k_{1}\left(k_{1}+1\right)}{\Omega_{1}}}\right)^{2 i_{1}+\mu_{1}-1} \frac{1}{i_{1} ! \Gamma\left(i_{1}+\mu_{1}\right)} \\
& \cdot \frac{2 \mu_{2}\left(k_{2}+1\right)^{\frac{\mu_{2}+1}{2}}}{\frac{\mu_{2}-1}{k_{2}} e^{k_{2} \mu_{2}} \Omega_{2}^{\frac{\mu_{2}+1}{2}}} \cdot \sum_{i_{2}=0}^{\infty}\left(\mu_{2} \sqrt{\frac{k_{2}\left(k_{2}+1\right)}{\Omega_{2}}}\right)^{2 i_{2}+\mu_{2}-1} \frac{1}{i_{2} ! \Gamma\left(i_{2}+\mu_{2}\right)} \\
& \cdot \int_{0}^{\infty} d y y^{-1-2 c_{1}+1+2 i_{1}+2 \mu_{1}-1} e^{-\frac{\mu_{1}\left(k_{1}+1\right)}{\Omega_{1}} y^{2}} \cdot \\
& \cdot \int_{0}^{\infty} d z \cdot z^{1+2 c_{1}-1+2 i_{2}+2 \mu_{2}-1} \cdot e^{-\frac{1}{\beta_{1}} \frac{w^{2} z^{2}}{y^{2}}-\frac{\mu_{2}\left(k_{2}+1\right)}{\Omega_{2}} z^{2}}
\end{aligned}
$$

Let us introduce the integral $J_{l}$ in the form:

$$
\begin{gathered}
J_{1}=\int_{0}^{\infty} d y y^{2 i_{1}+2 \mu_{1}-2 c_{1}-1} e^{-\frac{\mu_{1}\left(k_{1}+1\right)}{\Omega_{1}} y^{2}} . \\
\int_{0}^{\infty} d z \cdot z^{2 i_{2}+2 \mu_{2}+2 c_{1}-1} \cdot e^{-z^{2}\left(\frac{w^{2}}{\beta_{1} y^{2}}-\frac{\mu_{2}\left(k_{2}+1\right)}{\Omega_{2}}\right)}
\end{gathered}
$$

His solution is [14]: 


$$
\begin{gathered}
J_{1}=\int_{0}^{\infty} d y y^{2 i_{1}+2 \mu_{1}-2 c_{1}-1} e^{-\frac{\mu_{1}\left(k_{1}+1\right)}{\Omega_{1}} y^{2}} . \\
\frac{1}{2}\left(\beta_{1} \Omega_{2}\right)^{i_{2}+\mu_{2}+c_{1}} y^{2 i_{2}+2 \mu_{2}+2 c_{1}} \Gamma\left(i_{2}+\mu_{2}+c_{1}\right) \\
\frac{1}{\left(\Omega_{2} w^{2}+\mu_{2}\left(k_{2}+1\right) y^{2}\right)^{i_{2}+\mu_{2}+c_{1}}}= \\
=\frac{1}{2}\left(\beta_{1} \Omega_{2}\right)^{i_{2}+\mu_{2}+c_{1}} \Gamma\left(i_{2}+\mu_{2}+c_{1}\right) \cdot \\
\frac{1}{2} \cdot\left(\frac{\Omega_{2} w^{2}}{\mu_{2}\left(k_{2}+1\right)}\right)^{i_{1}+\mu_{1}+i_{2}+\mu_{2}} \frac{1}{\left(\Omega_{2} w^{2}\right)^{i_{2}+\mu_{2}+c_{1}}} \\
\int_{0}^{\infty} d t t^{i_{1}+\mu_{1}+i_{2}+\mu_{2}-1} \cdot \frac{1}{(1+t)^{i_{2}+\mu_{2}+c_{1}}} e^{-\frac{\mu_{1}\left(k_{1}+1\right)}{\Omega_{1}} \frac{\Omega_{2} w^{2}}{\mu_{2}\left(k_{2}+1\right)} t}
\end{gathered}
$$

By using the formula [14]:

$$
\int_{0}^{\infty} d s s^{a-1} \cdot e^{-c s} \cdot \frac{1}{(1+s)^{a+1-b}}=\Gamma(a) U(a, b, c),
$$

the integral $J_{l}$ obtains the form:

$$
\begin{gathered}
J_{1}=\frac{1}{2}\left(\beta_{1} \Omega_{2}\right)^{i_{2}+\mu_{2}+c_{1}} \Gamma\left(i_{2}+\mu_{2}+c_{1}\right) . \\
\frac{1}{2} \cdot\left(\frac{\Omega_{2}}{\mu_{2}\left(k_{2}+1\right)}\right)^{i_{1}+\mu_{1}+i_{2}+\mu_{2}} \frac{1}{\Omega_{2}{ }^{i_{2}+\mu_{2}+c_{1}}} w^{2 i_{1}+2 \mu_{1}-2 c_{1}} \cdot \Gamma\left(i_{1}+\mu_{1}+i_{2}+\mu_{2}\right) . \\
U\left(i_{1}+\mu_{1}+i_{2}+\mu_{2}, i_{1}+\mu_{1}-c_{1}, \frac{\mu_{1}\left(k_{1}+1\right) \Omega_{2}}{\mu_{2}\left(k_{2}+1\right) \Omega_{1}} w^{2}\right) \cdot
\end{gathered}
$$

B. Moments of Output Signal to Interference Ratio Moments of output signal to interference ratio are:

$$
\begin{gathered}
m_{n}=\overline{w^{n}}=\int_{0}^{\infty} d w w^{n} \cdot p_{w}(w)=\frac{2}{\Gamma\left(c_{1}\right) \beta_{1}^{c_{1}}} w^{2 c_{1}-1} . \\
\frac{2 \mu_{1}\left(k_{1}+1\right)^{\frac{\mu_{1}+1}{2}}}{k_{1}^{\frac{\mu_{1}-1}{2}} e^{k_{1} \mu_{1}} \Omega_{1}^{\frac{\mu_{1}+1}{2}}} \cdot \sum_{i_{1}=0}^{\infty}\left(\mu_{1} \sqrt{\frac{k_{1}\left(k_{1}+1\right)}{\Omega_{1}}}\right)^{2 i_{1}+\mu_{1}-1} \frac{1}{i_{1} ! \Gamma\left(i_{1}+\mu_{1}\right)} . \\
\frac{2 \mu_{2}\left(k_{2}+1\right)^{\frac{\mu_{2}+1}{2}}}{\frac{\mu_{2}-1}{k_{2}^{2}} e^{k_{2} \mu_{2}} \Omega_{2}^{\frac{\mu_{2}+1}{2}}} \cdot \sum_{i_{2}=0}^{\infty}\left(\mu_{2} \sqrt{\frac{k_{2}\left(k_{2}+1\right)}{\Omega_{2}}}\right)^{2 i_{2}+\mu_{2}-1} \frac{1}{i_{2} ! \Gamma\left(i_{2}+\mu_{2}\right)} . \\
\frac{1}{2}\left(\beta_{1} \Omega_{2}\right)^{i_{2}+\mu_{2}+c_{1}} \Gamma\left(i_{2}+\mu_{2}+c_{1}\right) \cdot \\
\Omega_{2}^{i_{1}+\mu_{1}-c_{1}} \\
\left(\mu_{2}\left(k_{2}+1\right)\right)^{i_{1}+\mu_{1}+i_{2}+\mu_{2}} \cdot \Gamma\left(i_{1}+\mu_{1}+i_{2}+\mu_{2}\right) \cdot \int_{0}^{\infty} d w w^{2 i_{1}+2 \mu_{1}-1+n} \\
U\left(i_{1}+\mu_{1}+i_{2}+\mu_{2}, i_{1}+\mu_{1}-c_{1}, \frac{\mu_{1}\left(k_{1}+1\right) \Omega_{2}}{\mu_{2}\left(k_{2}+1\right) \Omega_{1}} w^{2}\right)
\end{gathered}
$$

Let it be that integral $J_{2}$ is:

$$
\begin{gathered}
J_{2}=\frac{1}{2} \cdot\left(\frac{\Omega_{1} \mu_{2}\left(k_{2}+1\right)}{\Omega_{2} \mu_{1}\left(k_{1}+1\right)}\right)^{i_{1}+\mu_{1}+n / 2} \cdot \int_{0}^{\infty} d t t^{i_{1}+\mu_{1}-1+n / 2} \\
\cdot U\left(i_{1}+\mu_{1}+i_{2}+\mu_{2}, i_{1}+\mu_{1}-c_{1}, t\right)
\end{gathered}
$$

In the next step of the moment's derivation, we use the formula:

$$
\int_{0}^{\infty} d t t^{b-1} \cdot e^{-s t} U(a, c, t)=\frac{\Gamma(b) \Gamma(b+1-c)}{\Gamma(a+b+1-c)}
$$

$$
{ }_{2} F_{1}(b, b+1-c, a+b+1-c, 1-s) .
$$

For the considered case it is valid:

$$
\begin{gathered}
b=i_{1}+\mu_{1}+n / 2, \quad b+1-c=i_{1}+\mu_{1}+n / 2-i_{1}-i_{1}+c_{1}+1 \\
\quad a+b+1-c=i_{1}+\mu_{1}+i_{2}+\mu_{2}+n / 2+c_{1}+1, \quad s=0 \quad(15)
\end{gathered}
$$

After substituting, the expression for $J_{2}$ becomes:

$$
J_{2}=\frac{\Gamma\left(i_{1}+\mu_{1}+n / 2\right) \Gamma\left(n / 2+c_{1}+1\right)}{\Gamma\left(i_{1}+\mu_{1}+i_{2}+\mu_{2}+n / 2+c_{1}+1\right)}
$$

${ }_{2} F_{1}\left(i_{1}+\mu_{1}+n / 2, n / 2+c_{1}+1, i_{1}+\mu_{1}+i_{2}+\mu_{2}+n / 2+c_{1}+1,1\right)$

C. Moment Generating Function of Output Signal to Interference Ratio

Moment generating function of $w$ is:

$$
\begin{gathered}
M_{w}(s)=\overline{e^{s w}}=\int_{0}^{\infty} d w e^{s w} p_{w}(w)=\frac{2}{\Gamma\left(c_{1}\right) \beta_{1}^{c_{1}}} . \\
\cdot \frac{2 \mu_{1}\left(k_{1}+1\right)^{\frac{\mu_{1}+1}{2}}}{\frac{\mu_{1}-1}{k_{1}} e^{k_{1} \mu_{1}} \Omega_{1}^{\frac{\mu_{1}+1}{2}}} \cdot \sum_{i_{1}=0}^{\infty}\left(\mu_{1} \sqrt{\frac{k_{1}\left(k_{1}+1\right)}{\Omega_{1}}}\right)^{2 i_{1}+\mu_{1}-1} \frac{1}{i_{1} ! \Gamma\left(i_{1}+\mu_{1}\right)} \cdot \\
\cdot \frac{2 \mu_{2}\left(k_{2}+1\right)^{\frac{\mu_{2}+1}{2}}}{\frac{\mu_{2}-1}{k_{2}} e^{k_{2} \mu_{2}} \Omega_{2}^{\frac{\mu_{2}+1}{2}}} \cdot \sum_{i_{2}=0}^{\infty}\left(\mu_{2} \sqrt{\frac{k_{2}\left(k_{2}+1\right)}{\Omega_{2}}}\right)^{2 i_{2}+\mu_{2}-1} \frac{1}{i_{2} ! \Gamma\left(i_{2}+\mu_{2}\right)} . \\
\cdot \frac{1}{2}\left(\beta_{1} \Omega_{2}\right)^{i_{2}+\mu_{2}+c_{1}} \Gamma\left(i_{2}+\mu_{2}+c_{1}\right) \cdot \frac{1}{2} \cdot \frac{\Omega_{2}^{i_{1}+\mu_{1}-c_{1}}}{\left(\mu_{2}\left(k_{2}+1\right)\right)^{i_{1}+\mu_{1}+i_{2}+\mu_{2}}} . \\
\left.\cdot U\left(i_{1}+\mu_{1}+i_{2}+\mu_{2}\right) \cdot \sum_{k=0}^{\infty} \frac{s^{k_{1}}}{k_{1} !} \int_{0}^{\infty} d w w^{2 i_{1}+2 \mu_{1}-1+k_{1}} . i_{2}+\mu_{2}, i_{1}+\mu_{1}-c_{1}, \frac{\mu_{1}\left(k_{1}+1\right) \Omega_{2}}{\mu_{2}\left(k_{2}+1\right) \Omega_{1}} w^{2}\right)
\end{gathered}
$$

Let there be integral $J_{3}$ :

$$
\begin{gathered}
J_{3}=\int_{0}^{\infty} d w w^{2 i_{1}+2 \mu_{1}-1+k_{1}} . \\
U\left(i_{1}+\mu_{1}+i_{2}+\mu_{2}, i_{1}+\mu_{1}-c_{1}, \frac{\mu_{1}\left(k_{1}+1\right) \Omega_{2}}{\mu_{2}\left(k_{2}+1\right) \Omega_{1}} w^{2}\right)
\end{gathered}
$$

The integral $J_{3}$ be solved as:

$$
\begin{aligned}
& J_{3}= \frac{1}{2} \cdot\left(\frac{\Omega_{1} \mu_{2}\left(k_{2}+1\right)}{\Omega_{2} \mu_{1}\left(k_{1}+1\right)}\right)^{i_{1}+\mu_{1}+k / 2} \cdot \int_{0}^{\infty} d t t^{i_{1}+\mu_{1}+k / 2-1} \\
& U\left(i_{1}+\mu_{1}+i_{2}+\mu_{2}, i_{1}+\mu_{1}-c_{1}, t\right)= \\
&= \frac{\Gamma\left(i_{1}+\mu_{1}+k / 2\right) \Gamma\left(k / 2+c_{1}+1\right)}{\Gamma\left(i_{1}+\mu_{1}+i_{2}+\mu_{2}+k / 2+c_{1}+1\right)} \\
&{ }_{2} F_{1}\left(i_{1}+\mu_{1}+k / 2, k / 2+c_{1}+1, i_{1}+\mu_{1}+i_{2}+\mu_{2}+k / 2+c_{1}+1,1\right) \\
& \frac{1}{2} \cdot\left(\frac{\Omega_{1} \mu_{2}\left(k_{2}+1\right)}{\Omega_{2} \mu_{1}\left(k_{1}+1\right)}\right)^{i_{1}+\mu_{1}+k / 2}
\end{aligned}
$$

III. NUMERICAL RESUlTS

The first moment of $w$ (mean value $\bar{w}$ ) is given versus Rician factor of desired signal $k_{l}$, Rician factor of interference signal $k_{2}$ and Gamma long term shape parameter $\beta$ in the next few figures, with different parameters of curves, such as Gamma large scale severity parameter $c$ and parameter $\mu$. 
In Fig. 1, the main value $\bar{w}$ versus Rician factor of desired signal $k_{l}$ is presented for $\beta=0.2, \mu=2$, and $c=2$; Rician factor of interference signal $k_{2}$ is variable. In Fig. 2, the first moment $\bar{w}$ is plotted versus Rician factor of desired signal $k_{l}$. The parameters of curves are: Rician factor of interference signal $k_{2}=0.2, \mu=2$ and $c=2$; parameter $\beta$ is changeable. One can see from Fig. 1 that the first moment of $w$ increases when Rician factor of desired signal, $k_{l}$, increases. The first moment also is growing up with declining of Rician factor of interference signal $k_{2}$. An increment of mean value of $w$ is visible from Fig. 2 for reduction of parameter $\beta$, whereby the increase is more conspicuous for larger values of Rician factor of desired signal $k_{l}$.

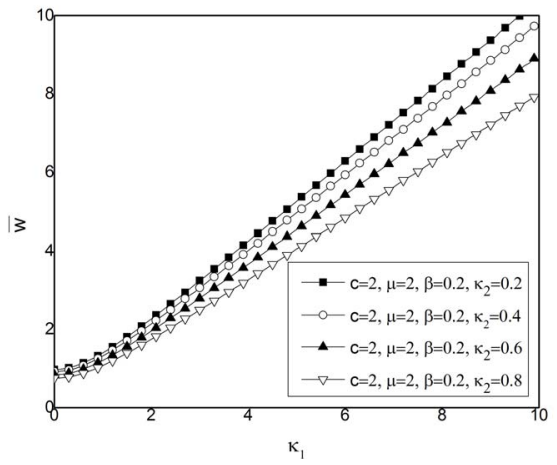

Figure 1. Mean value $\bar{w}$ versus Rician factor of desired signal $k_{l}$ for $\beta=0.2, \mu=2, c=2$ and variable Rician factor of interference signal $k_{2}$.

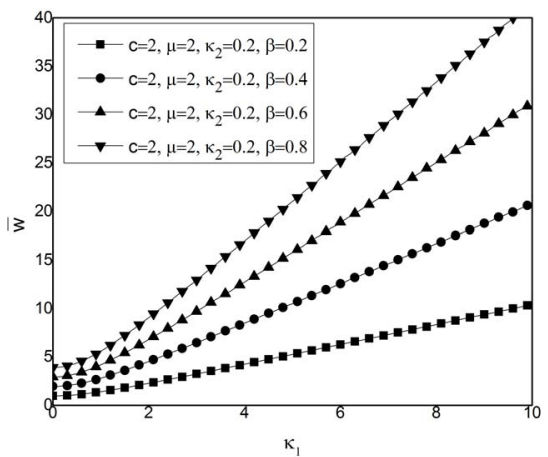

Figure 2. The first moment of $w$ versus Rician factor of desired signal $k_{1}$ for $c=2, \mu=2, k_{2}=0.2$, and changeable parameter $\beta$.

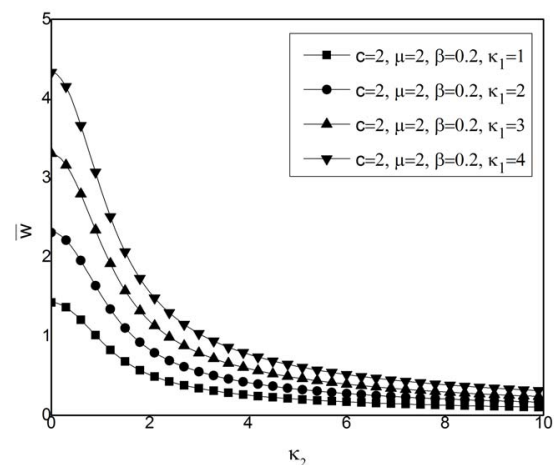

Figure 3. Mean value $\bar{w}$ versus Rician factor of interference signal $k_{2}$ for $c=2, \mu=2, \beta=0.2$ and changeable Rician factor of desired signal $k_{l}$.
Mean value $\bar{w}$ versus Rician factor of interference signal $k_{2}$ is shown in Figs. 3 and 4. In Fig. 3, the parameters of curves are: $c=2, \mu=2, \beta=0.2$ and modifiable Rician factor of desired signal $k_{l}$. In Fig. 4, the curves are drawn for: $c=2, \mu=2, k_{l}=1$ and variable parameter $\beta$. It is evident from these figures that the mean value wanes with increasing of Rician factor of interference signal $k_{2}$, increases with increasing of Rician factor of desired signal $k_{1}$ and rises with bigger parameter $\beta$. For smaller values of Rician factor $k_{2}$, the influence of Rician factor $k_{l}$ and parameter $\beta$ is larger.

The first moment of $w$ versus parameter $\beta$ is presented in Figs. 5. and 6. Gamma shadowing severity parameter $c$ and $k$ - $\mu$ distribution parameter $\mu$ are equal for all observed cases: $c=2, \mu=2$.

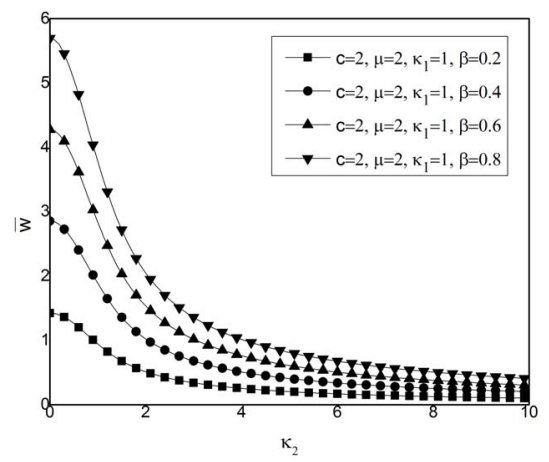

Figure 4. Mean value $\bar{w}$ versus Rician factor of interference signal $k_{2}$ for $c=2, \mu=2, k_{1}=1$ and variable parameter $\beta$.

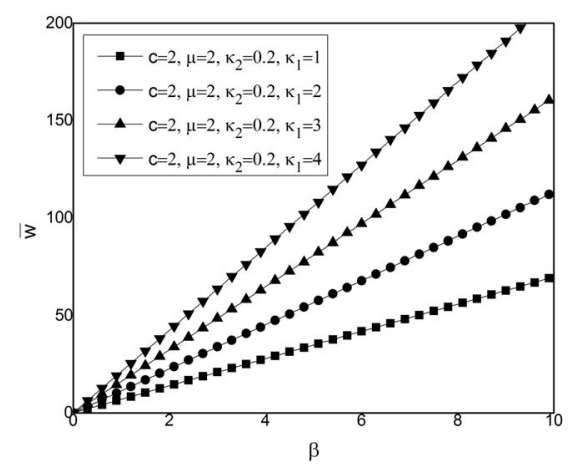

Figure 5. Mean value $\bar{w}$ versus parameter $\beta$ for $c=2, \mu=2$, Rician factor of interference signal $k_{2}=0.2$, and variable Rician factor of desired signal $k_{l}$.

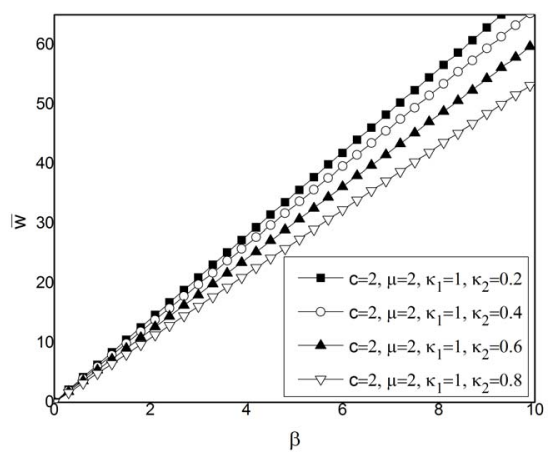

Figure 6. The first moment of $w$ versus parameter $\beta$ for $c=2, \mu=2$, Rician factor of desired signal $k_{l}=1$ and changeable Rician factor of interference signal $k_{2}$. 


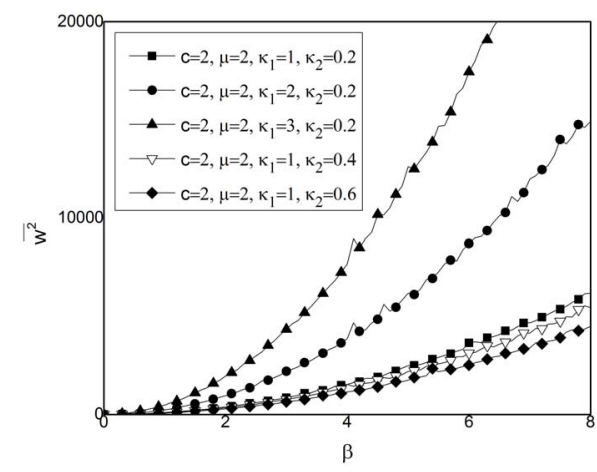

Figure 7. The second moment of $w$ versus parameter $\beta$ with parameters $c=2$ and $\mu=2$, and variable Rician factor of desired signal $k_{1}$ and Rician factor of interference signal $k_{2}$.

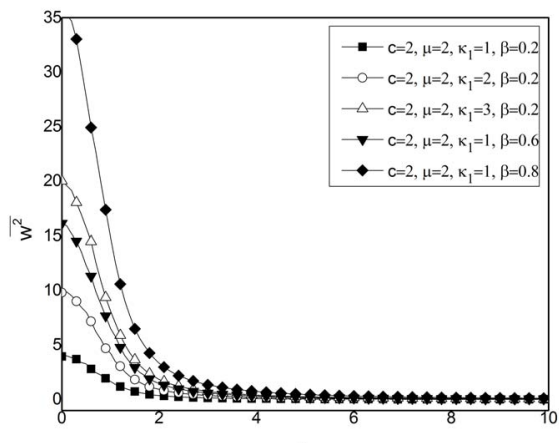

$\kappa_{2}$

Figure 8. The squared average value $\overline{w^{2}}$ versus Rician factor of interference signal $k_{2}$ for $c=2, \mu=2$, and changeable Rician factor of desired signal $k_{l}$ and parameter $\beta$.

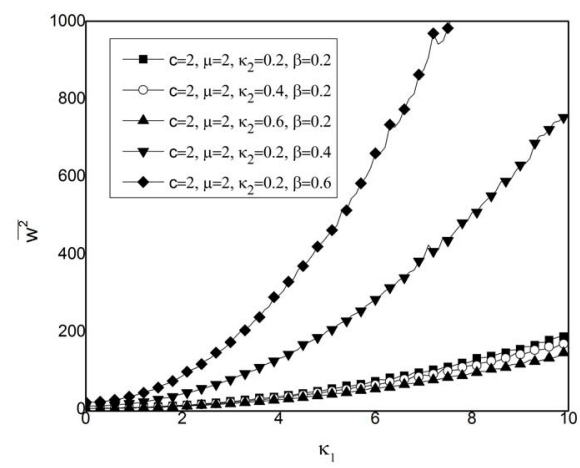

Figure 9. The second moment $\overline{w^{2}}$ versus Rician factor of desired signal $k_{1}$ for $c=2, \mu=2$ and mutable Rician factor of interference $k_{2}$ and parameter $\beta$.

The Rician factor of desired signal $k_{l}$ is changeable in Fig. 5. and Rician factor of interference signal $k_{2}$ is varying in Fig. 6. It is obvious from these two figures that mean value $\bar{w}$ is growing with an enlargement of parameter $\beta$. One can also see that influence of Rician factors $k_{1}$ and $k_{2}$ is bigger for larger values of parameter $\beta$.

The second moment of $w$ i.e. squared average value $\overline{w^{2}}$, is given versus parameter $\beta$, Rician factor of interference signal $k_{2}$ and Rician factor of desired signal $k_{l}$, in Figs. 7 to 9, respectively. The parameters of curves are Gamma shadowing severity parameter $c$ and parameter $\mu: c=2, \mu=2$, as well as Rician factor of desired signal $k_{1}$, Rician factor of interference signal $k_{2}$ and parameter $\beta$. It is obvious from last three figures that second moment $\overline{w^{2}}$ enhances with enlargement of parameter $\beta$ and Rician factor of desired signal $k_{l}$.

The squared average value decreases with increasing of Rician factor of interference signal $k_{2}$. The greatest impact on increasing of the second moment of $w$ has increment of parameter $\beta$. The influence of parameter $\beta$ is bigger for higher values of Rician factor of desired signal $k_{l}$ and vice versa. For bigger values of Rician factor of desired signal $k_{l}$, the impact of increase of Rician factor of interference $k_{2}$ is negligible.

\section{CONCLUSION}

Wireless communication system operating over $\mathrm{k}-\mu$ multipath fading channel in the presence of cochannel interference subjected to shadowed short term fading is considered here. Desired signal experiances $\mathrm{k}-\mu$ multipath fading resulting in signal envelope variation. Cochannel interference expiriences Gamma long term fading resulting in signal envelope average power variation and also $k-\mu$ short term fading resulting in signal envelope variation.

In interference limited environments, ratio of signal envelope to interference envelope is important performance measure which for proposed model can be calculated as the ratio of product of square rooted Gamma random variable and the $\mathrm{k}-\mu$ random variable and $\mathrm{k}-\mu$ random variable. The closed form expressions for probability density function, moments and moment generating function of signal to interference ratio at output are derived.

From obtained expressions it is possible to evaluate probability density function, moments and moment generating function of signal to interference ratio at the output of wireless communication system in the presence of Nakagami- $m$ short term fading and cochannel interference subjected to Gamma long term fading and Nakagami- $m$ short term fading [15].

System performance is better for higher values of the first moment and the second moment. The first moment increases as Rician factor of desired signal increases. The impact of Rician factor of desired signal, Rician factor of cochannel interference and Gamma long term severity parameter on the first moment and the second moment are analyzed and discussed.

\section{ACKNOWLEDGMENT}

This paper has been partially funded by the Ministry of Education, Science and Technological Development of Republic of Serbia under projects TR33035 and III-44006.

\section{REFERENCES}

[1] M. K. Simon, M. S. Alouini, Digital Communication over Fading Channels. USA: John Wiley \& Sons, 2000. 
[2] S. Panic, M. Stefanović, J. Anastasov, P. Spalevic, Fading and Interference Mitigation in Wireless Communications. USA: CRC Press, 2013.

[3] P. M. Shankar, Fading and Shadowing in Wireless Systems, Springer, Dec 7, 2011. DOI 10.1007/978-1-4614-0367-8

[4] M.D. Yacoub, "The k- $\mu$ distribution", http://www.eletrica.ufpr.br/anais/sbrt/SBrT19/001000000008 700059.pdf

[5] S. L. Cotton, W. G. Scanlon, "Higher-order statistics for k- $\mu$ distribution", Electronics Letters, 43(22), no 2, 2007, pp. 1215-1217, DOI:10.1049/el:20072372

[6] A. D. Cvetkovic, M. C. Stefanovic, N. M. Sekulovic, D. N. Milic , D. M. Stefanovic, Z.J. Popovic, “ Second-order statistics of dual SC macrodiversity system over channels affected by Nakagami-m fading and correlated gamma shadowing”, Przegląd Elektrotechniczny (Electrical Review), ISSN 0033-2097, R. 87 NR 6/2011, pp. 284-288.

[7] N.M. Sekulovic, M. Č. Stefanovic, "Performance Analysis of System with Micro- and Macrodiversity Reception in Correlated Gamma Shadowed Rician Fading Channels", Wireless Personal Communications, July 2012, Volume 65, Issue 1, pp 143-156, First online: 12 February 2011, http://www.hindawi.com/journals/ijdmb/2009/573404/

[8] S. R. Panic, D. M. Stefanovic, I. M. Petrovic, M. C. Stefanovic, J. A. Anastasov, D S. Krstic, "Second order statistics of selection macro-diversity system operating over Gamma shadowed k- $\mu$ fading channels", EURASIP Journal on Wireless Communications and Networking, 2011:151, Article ID 631204, doi:10.1186/1687-1499-2011-151

[9] M. Milisic, M. Hamza, M. Hadzialic, "BEP/SEP and Outage Performance Analysis of L BranchMaximal-Ratio Combiner for $\kappa-\mu$ Fading", International Journal of Digital Multimedia Broadcasting, Vol. 2009, Article I 573404, pp. 1-8. 13.

[10] M. C. Stefanovic, D. M. Milovic, A. M. Mitic, M. M. Jakovljevic, "Performance analysis of system with selection combining over correlated Weibull fading channels in the presence of cochannel interference", AEU - International Journal of Electronics and Communications, Vol. 62, Issue 9, October 2008, pp. 695-700.

[11] D. N. Milic, D. B. Đošic, C. M. Stefanovic, M. M. Smilic, S. N. Suljovic, "Outage performance of multi-branch SC receiver over correlated Weibull channel in the presence of correlated Rayleigh co-channel interference", Facta Universitatis, Series: Automatic Control and Robotics, Vol. 14, No 3, 2015, pp. $183-191$.

[12] A. S. Panajotovic, N. M. Sekulovic, M. C. Stefanovic, D. Lj. Draca, "Average Level Crossing Rate of Dual Selection Diversity over Correlated Unbalanced Nakagami-m Fading Channels in the Presence of Cochannel Interference", IEEE Communications Letters 16(5), 2012, pp. 691-693.

[13] T.S. Rappaport, Wireless Communications: Principles and Practice, Prentice-Hall, New York, 1996.

[14] Gradshteyn I. and Ryzhik I., Tables of Integrals, Series, and products, Academic Press, New Yourk, 1st edition, 1980.

[15] D. Krstić, Z. Jovanović, R. Gerov, M. Stefanovic, M.Gligorijević, "Performance Analysis of Wireless Communication System in the Presence of Gamma Shadowing, Nakagami-m Multipath Fading and Cochannel Interference", International Journal of Communications, 1, 2016, pp. 150-157. 